\title{
Yoked Versus Self-Controlled Practice Schedules and Performance on Dual-Task Transfer Tests
}

\author{
Elizabeth A. Sanli ${ }^{*}$ and Timothy D. Lee
}

Department of Kinesiology, McMaster University, 1280 Main Street West, Hamilton, ON L8S 4K1, Canada

\begin{abstract}
The authors examined yoked versus self-controlled practice schedules to determine their influence in immediate and delayed dual-task performance. The task was to propel a small disc along a smooth table top, with the purpose of stopping it in a specified target area. Participants in the self-controlled schedule group chose the order in which eight acquisition targets, differing in distance from a home position, were practiced during acquisition. Members of a control group followed identical schedules to yoked participants in the self-controlled group. The authors hypothesized that those in the self-controlled group would perform with less error on retention and transfer tests and with more error on dual-task transfer tests in comparison to those in the yoked group. No differences in performance on retention, transfer, or dual-task tests were found. Possible reasons for the similar performance between groups include the provision of choice over blocks of rather than individual trials and feelings of autonomy in both groups due to choice as to how to propel the disc.
\end{abstract}

Keywords: Choice, Cognitive Effort, Explicit, Implicit, Progression, Secondary Task.

\section{INTRODUCTION}

The optimization of practice scheduling and organization for the acquisition and learning of motor skills can be of benefit in many contexts, such as teaching, coaching, and rehabilitation. Some recent studies (see $[1,2]$ for reviews) have examined the utility of providing a learner with control over a portion of their practice context, including the scheduling of practice (e.g. [3]). This provision of control over a portion of the practice context to the learner is referred to as self-controlled practice [1]. Currently there are two categories of possible explanations for the benefits of self-control in a motor-learning context. One category of explanation hypothesizes that self-controlled practice plays a role in the motivation of the learner (see [4] for review), while the other category hypothesizes that one possible reason why a robust benefit of self control is seen in comparison to those not provided control over practice (yoked groups) is that those in a self-controlled condition engage in more cognitive effort than those in a yoked condition. In particular, it is hypothesized that those provided choice over a portion of practice, such as the scheduling of tasks, have greater demands placed on cognitive processes involved in decision making, monitoring, evaluating, correcting and strategizing $[3,5]$. These categories of explanation are not contradictory, and may, in fact, complement each other. However no single theoretical perspective in the current motor-learning literature has been proposed to address the robust benefit of the provision of self-control during practice of a motor task.

*Address correspondence to this author at the Department of Kinesiology, McMaster University, 1280 Main Street West, Hamilton, ON L8S 4K1, Canada; Fax: 905-523-6011;

E-mail: sanliea@mcmaster.ca
Benefits to learning have been reported for the provision of self-control over a number of aspects of practice scheduling. For example, self-control of the order in which versions of a task are practiced, $[3,6]$ the progression though increasingly difficult versions of a task, [7] variability of practice, [5] and when to cease practice [8-10] have all been found to contribute beneficially to learning.

While in the self-controlled practice literature, discussed above (see $[1,2]$ for reviews), cognitive effort in acquisition is presented as beneficial, the implicit learning literature suggests a detrimental effect of cognitive effort during acquisition on later performance under dual-task conditions. Research indicates that, under specific conditions, the initial learning of a motor task can effectively bypass the earlier cognition-intensive stages of the learning process and in doing so minimize the amount of knowledge that can inappropriately reappear in later stages of learning, especially when attention demands are put under pressure [11]. This is referred to as implicit [11] or U-mode [12] learning. Implicit knowledge is described as knowing without awareness or the ability to articulate knowledge whereas explicit knowledge is made up of information (e.g. facts and rules) of which we are aware and thus can articulate.

Baddeley and Wilson [13] proposed that explicit processes are required for error detection and elimination, whereas errors are unable to be corrected in situations without explicit knowledge such as in implicit learning. Because of these differences, the performance-supporting knowledge base of implicit learners contains a greater number of error experiences in comparison to explicit learners because the errors cannot be identified and filtered out. This provides a possible explanation for a benefit of the minimization of errors in implicit learning [13, 14]. Maxwell, 


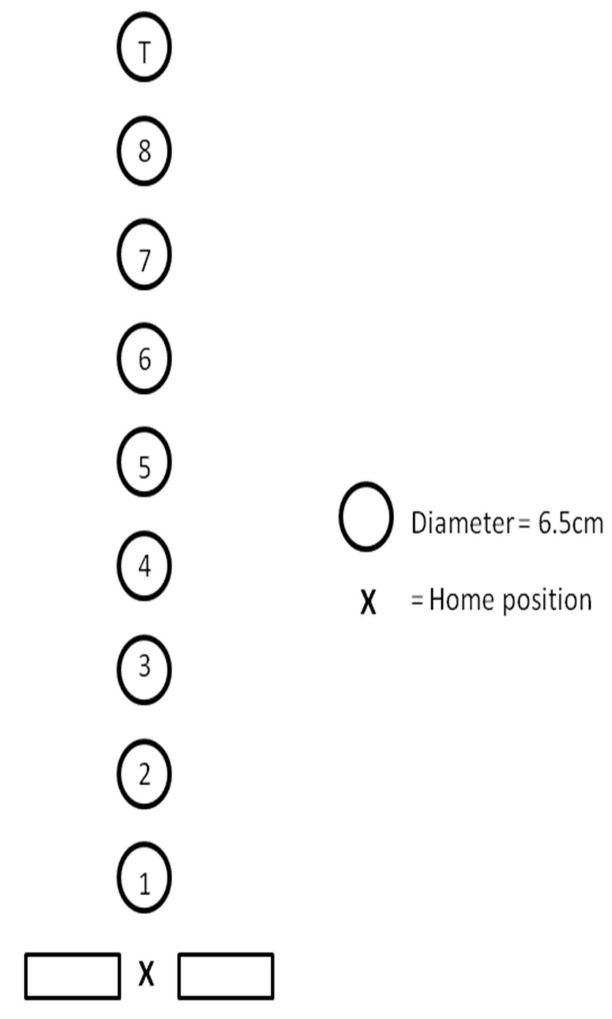

Fig. (1). Diagram of the location of each of the acquisition and transfer targets in relation to the home position. During the experiment only one target was displayed at a time. Each of the targets is numbered and is referred to as such in the text.

Masters, Kerr and Weedon [14] further suggested that a more implicit form of learning will occur when no (or little) hypothesis testing (involving explicit learning) is required during acquisition. One implication of this suggestion is that practice schedules that encourage cognitive effort, such as a self-controlled practice schedule, may be detrimental to later performance under dual-task transfer conditions, whereas a yoked schedule, with less cognitive effort may produce better performance when specifically tested under dual-task transfer.

The influence of self-controlled versus yoked practice on learning a novel task is typically measured using retention tests, transfer tests or both. Evidence is mixed as to whether implicit learning is beneficial for performance during acquisition, retention and transfer. However, the literature has consistently shown that those who learn a task under implicit practice conditions outperform those under an explicit condition when tested under attention-demanding, dual-task transfer conditions. Therefore, the purpose of the present experiment was to determine if self-controlled and yoked practice schedules would elicit differences in a dualtask transfer test - an experimental prediction that has previously not been tested. The implicit learning literature suggests that the characteristics of cognitive effort, such as that which takes place in self-controlled learning contexts, hinder performance on dual-task tests, therefore we hypothesize that: 1) based upon previous comparisons of self-controlled and yoked scheduling of practice (e.g. [3]), no differences would be seen between groups during the acquisition session for any of the dependent variables, 2) that the self-controlled practice group would perform with less error, 2-dimensional centroid error (CE) and 2-dimensional variable error (VE) than the yoked practice group on retention and transfer tests and 3) based upon previous comparisons of greater and lesser amounts of cognitive effort in practice (e.g. [14]) that the yoked group would perform with less (CE) and (VE) than the self-controlled group on the dual-task tests.

\section{METHODS}

\section{Participants}

Twenty young adults from a university and surrounding community participated in the study (10 females and 10 males, $M$ age $=21.8$ years, $S D=4.2$ ). All but one female participant reported a preference to use their right hand. This research was approved by the institutional review board and participants were unaware of the purposes of the experiment. Participants did not have previous experience with the experimental task. Informed consent was obtained from all participants. Participants were alternately assigned to the self-controlled or yoked group based on gender such that the first female participant was assigned to the self-controlled group while the second female participant was assigned to the yoked group and the first male to the self group and second male to the yoked group, etc. Self- and yoked-pairs were male to male and female to female.

\section{Task}

The participants were required to propel a small disc along a smooth table top, with the purpose of stopping the disc in a specified target area (a lighted circle projected onto the surface of the table). Participants could choose to propel the disc however they wished; using their preferred hand, as long as the disc was released at the home position and slid along the table top (e.g., did not become airborne or break contact with the table surface).

\section{Apparatus}

Participants were seated at a table, $69.5 \mathrm{~cm}$ high, with a smooth table top measuring $76.5 \mathrm{~cm} \times 92 \mathrm{~cm}$. The home position was in the center of the table-top, $9 \mathrm{~cm}$ from the edge closest to the participant and was indicated with a small red X. White tape was placed $2.5 \mathrm{~cm}$ from either side of the $\mathrm{X}$ to further encourage proper positioning of a $3 \mathrm{~cm}$ diameter, brown, plastic disc at the start of each trial. An $11 \mathrm{~mm}$ infrared emitting diode (IRED) was glued to the center of the disc. The weight of the combined disc and IRED was $12.5 \mathrm{~g}$. The IRED was attached to an Optotrak 3020 that collected three-dimensional data, sampled at $500 \mathrm{~Hz}$. An Epson PowerLite 50c projector was suspended $109 \mathrm{~cm}$ from the table top and connected to a computer. Microsoft Power Point was used to project a $6.5 \mathrm{~cm}$ diameter, white circle onto the table top. The target was displayed as a white circle with a surrounding black background. Nine targets were used in the experiment with the front edge of the target located 3.5, $7.5,11.5,15.5,18.5,22.5,26.5,30.5$ or $34.5 \mathrm{~cm}$ from the home position (the red X - see Fig. (1) for a diagram of the targets used). The order in which the targets were presented during acquisition differed by experimental group. For the 
dual-task tests, a customizable software program (E-prime version 1.2, Psychology Software Tools Inc., Pittsburgh, PA) presented $500 \mathrm{~Hz}$ or $1000 \mathrm{~Hz}$ auditory tones at intervals of $1500 \mathrm{~ms}$. The tones were delivered by two speakers placed $60 \mathrm{~cm}$ from the participant.

\section{Procedure}

An experimenter read a script that described the task to participants in both groups. The two groups were given similar instructions, with the exception that participants in the self-controlled group were told that they would choose the order in which the targets were practiced while the participants in the yoked group were told that the experimenter would choose the order. Both groups were shown the target that would be used for retention as well as a diagram of each of the acquisition targets prior to the beginning of acquisition. Those in the self-controlled group chose the target to which they aimed for each block of trials, with restrictions that each target was to be used for one block of trials and that all targets must be used during acquisition. Those in the yoked group were informed of the target that was to be used for the upcoming block of trials, that each target would only be used once and that all targets would be used during acquisition. The order of targets for the yoked participants followed a schedule chosen by a counterpart in the self-controlled group. In total, the participants completed 200 acquisition trials ( 25 to each of 8 targets), 50 retention trials (25 immediate and 25 delayed), 50 dual-task trials (25 immediate and 25 delayed), and 50 transfer trials (25 immediate and 25 delayed).

Acquisition began with the instructions; the participants then chose (or were told) which target would be used first on a diagram of all the possible targets. The target chosen then was filled in with the number 1 on the diagram to indicate that it was the first target chosen (or assigned). When participants were ready to begin they placed the disc on the home position and propelled the disc towards the appropriately-lit target (the other targets were not visible). The experimenter then recorded the end location of the disc using Optotrak. The experimenter then raised her hand indicating to return the disc to the home position and begin the next trial. Participants completed 25 trials at each target before choosing or being assigned the next target. At the completion of acquisition participants were asked to work on a "Hard Sodoku Puzzle" (from http://www.sudokupuzz.com) for 10 minutes.

Following the puzzle, participants completed a retention test consisting of 25 trials at target 8 (the farthest acquisition target.) This was followed by a dual-task test, consisting of 25 trials which also used target 8, with the added task of counting the total number of high tones $(1000 \mathrm{~Hz})$ that were presented in the random series of high and low $(500 \mathrm{~Hz})$ beeps throughout the entire block of trials. Participants were asked to report the total number of high beeps at the conclusion of the series. Participants then completed an additional 25 trials to target 9 which had not been previously practiced (the transfer test). Participants returned one day later for delayed retention, dual-task and transfer tests, which were identical to the immediate tests.

\section{Data Analyses}

The dependent variables; proportion of errors per block, two-dimensional centroid error (CE) and variable error (VE) were used to examine performance during acquisition, retention, dual-task and transfer tests. Proportion of errors was defined as the proportion of the total trials, within a block (of 25 trials) where the disc did not land completely within the lighted target. While the proportion of errors per block provides a global measure of task success, twodimensional $\mathrm{CE}$ and $\mathrm{VE}$ provide measures of the average magnitude and variability of end-location. $\mathrm{CE}$ is a measure of the magnitude of bias from the target centre over a block of trials and VE is a measure of variability relative to the individual's centroid within each block. Both of these measures take into account the two-dimensional nature of the task and are independent of the axes chosen to record the raw data [15].

For acquisition, all three dependent variables were analyzed using separate 2 (groups: Self-controlled, Yoked) x 8 (targets) analysis of variance (ANOVAs) with repeated measures on target. We chose to analyze acquisition by comparing targets rather than blocks of trials because each pair of participants used the targets in a unique order. For example, for block one; every single target may have been used by one or more participants in each of the groups, making comparison between the groups on a block by block basis relatively meaningless. Comparing performance on the same target, regardless of the block in which it was practiced, provides a more meaningful comparison as the same number of participants in each group would have practiced that target at some point during acquisition.

In order to gain insight into pattern order strategy, we examined the pattern of target choices made by those in the self-controlled group. Participants that chose to follow a progressive pattern, starting at target 1 and ending at target 8 , with one or less deviations were labeled with a progressive pattern $(n=5)$. Participants that chose two or more deviations from a progressive pattern were labeled with a random pattern $(n=5)$. In acquisition, all three dependent variables were analyzed using separate 2 (pattern order: progressive, random) x 2 (groups: Self-controlled, Yoked) x 8 (targets) analysis of variance (ANOVAs) with repeated measures on target.

For immediate and delayed retention, dual-task and transfer tests, the dependent variables were analyzed using separate 2 (group: Self-controlled, Yoked) x 2 (sessions: immediate, delayed) x 3 (tests: retention, dual-task, transfer) ANOVAs with repeated measures on both session and test. To determine if differences in pattern strategy played a role in learning, the dependent variables were analyzed using separate 2 (pattern order: progressive, random) x 2 (group: Self-controlled, Yoked) x 2 (sessions: immediate, delayed) $\mathrm{x}$ 3 (tests: retention, dual-task, transfer) ANOVAs with repeated measures on both session and test. In order to determine if participants experienced a change in performance from acquisition to immediate and delayed tests, separate 2 (groups: Self-controlled, Yoked) $\times 3$ (blocks: acquisition, immediate test, delayed test) ANOVAs were conducted for all three dependent variables for both retention and dual-tasks tests. This analysis was not 


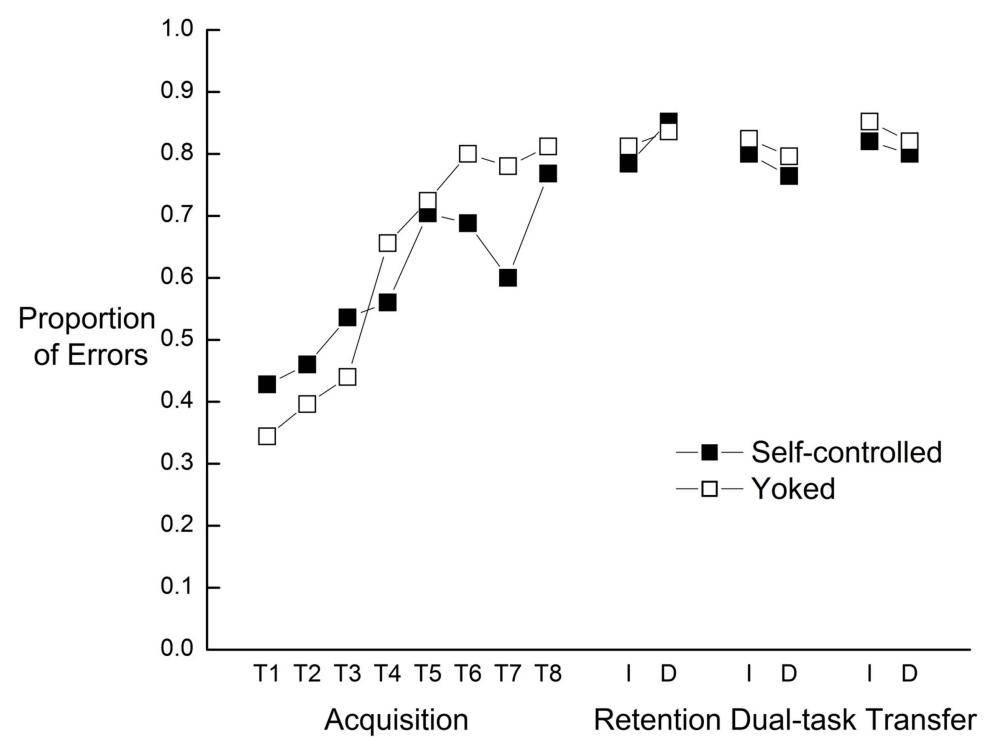

Fig. (2). Proportion of trials in each block of 25 trials, where the disc did not land within the target for targets one through eight in acquisition as well as immediate and delayed retention, dual-task and transfer tests.

performed for the transfer tests as the target for the tests was not used in acquisition. To examine performance on the counting portion of the dual-task test a 2 (group; selfcontrolled, yoked) $\mathrm{x}$ 2(session; immediate, delayed) ANOVA with repeated measures on session was conducted. Tukey's Honestly Significant Difference (HSD) tests were used to contrast mean differences where appropriate. Alpha was set at 0.05 for all statistical analyses.

\section{RESULTS}

\section{Acquisition}

For the proportion of errors dependent measure, a main effect was revealed for Target $[F(7,126)=23.79, p<.001]$, which generally showed that errors increased for targets located farther from the home position. Specifically, the post-hoc tests revealed that target 8 elicited significantly more errors than targets 1-4; targets 5, 6 and 7 elicited significantly more errors than targets $1-3$ and the target 4 elicited significantly more errors than targets 1 and 2. Posthoc analyses of a significant interaction between target and group $[F(7,126)=2.57, p=.017]$ revealed that for targets 6 and 7 , those in the yoked condition performed with significantly more errors than those in the self-controlled condition. There was no significant main effect revealed for group (see the left side of Fig. 2).

For CE, a main effect was revealed for target $[F(7,126)$ $=4.31, p<.001]$, with post-hoc test revealing that target 8 elicited significantly greater $\mathrm{CE}$ than targets $1-5$. No significant main effect for group or interaction between target and group were revealed.

For VE, a main effect was revealed for target $[F(7,126)=$ $28.63, p<.001]$, with post-hoc tests revealing that target 8 elicited significantly more variability than target 7 as well as targets 1-5. Targets 6 and 7 elicited significantly more variability than targets $1-4$, target 5 elicited significantly more variability than targets $1-3$, and target 4 elicited significantly more variability than targets 1 and 2 . No significant main effect for group or interaction between target and group were revealed.

In the examination of pattern order strategy it was found that 3 participants chose a progressive schedule, 2 chose a schedule with one deviation from progressive and 5 chose a schedule with more than one deviation from progressive.

A main effect for pattern of order $[F(1,16)=5.00, p=$ $.04]$ revealed that those who followed a random pattern of targets, regardless of group, performed with less proportion of error in acquisition $(M=.54, S D=.14)$ than those who followed a progressive pattern of targets $(M=.67 S D=.17)$.

For means and standard deviations for acquisition please see Table 1.

\section{Immediate and Delayed Tests}

No significant main effects or interactions were found for retention, dual-task or transfer tests on any of the dependent variables. No significant differences on any of the dependent variables between performance in acquisition and immediate or delayed retention or dual-task tests were revealed (see the right side of Fig. 2). Also, there were no significant differences in dual-task counting performance between groups or testing sessions. For means and standard deviations for tests of learning please see Tables $\mathbf{2 , 3}$ and $\mathbf{4}$.

\section{DISCUSSION}

\section{Measures of Performance}

Using different distances from the home position to the target was effective in manipulating task difficulty, as revealed by the increase in error with increased distance 
Table 1. Means and Standard Deviations for Proportion of Errors, CE and VE During Acquisition

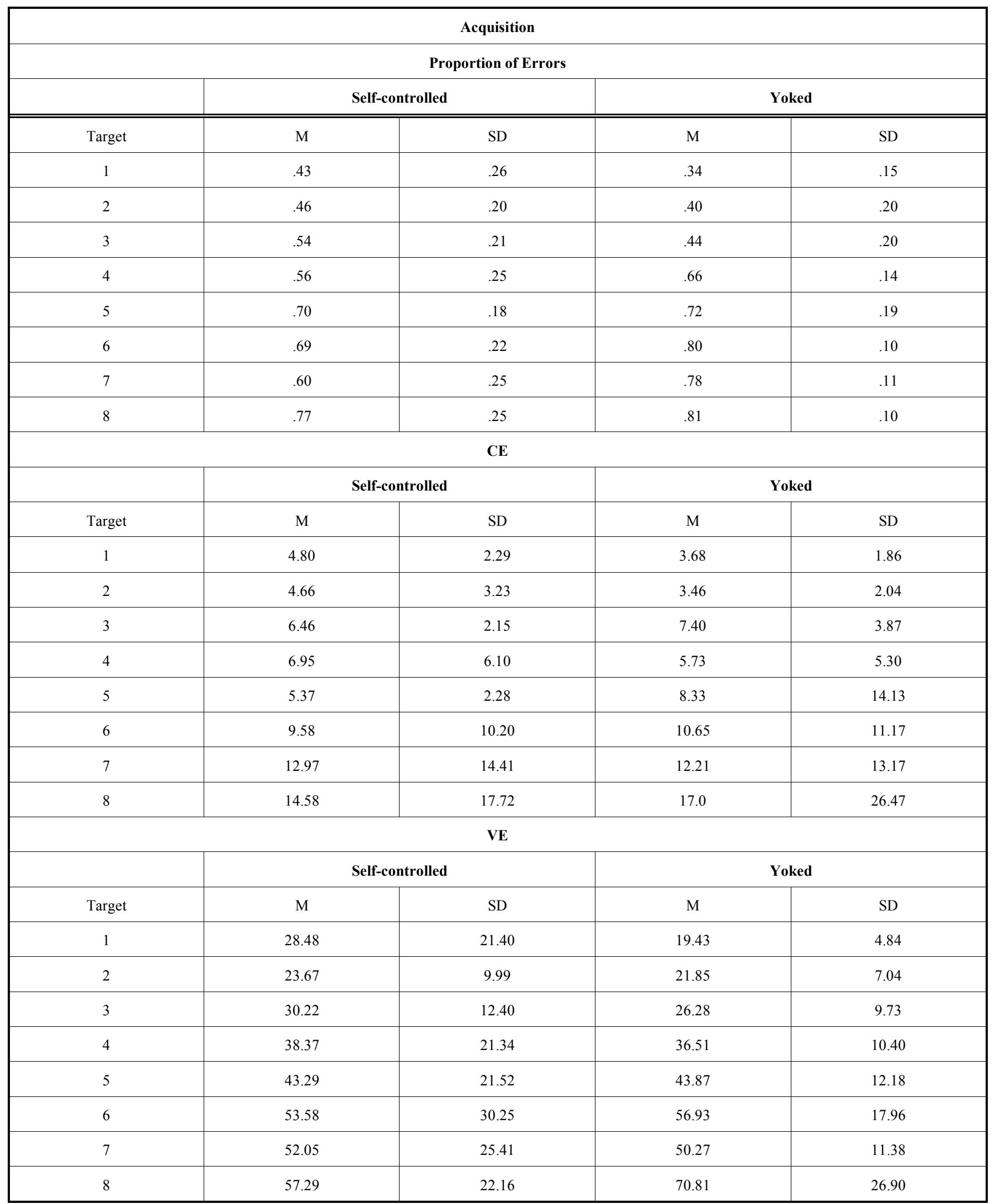


Table 2. Means and Standard Deviations for Proportion of Errors, CE and VE for Immediate and Delayed Retention Tests

\begin{tabular}{|c|c|c|c|c|}
\hline \multicolumn{5}{|c|}{ Retention } \\
\hline \multirow[b]{2}{*}{ Test } & \multicolumn{2}{|c|}{ Self-Controlled } & \multicolumn{2}{|c|}{ Yoked } \\
\hline & $\mathbf{M}$ & SD & $\mathbf{M}$ & SD \\
\hline \multicolumn{5}{|c|}{ Proportion of Errors } \\
\hline I & .78 & .20 & .81 & .09 \\
\hline \multicolumn{5}{|c|}{$\mathbf{C E}$} \\
\hline I & 18.83 & 17.08 & 19.11 & 17.30 \\
\hline $\mathrm{D}$ & 21.98 & 16.76 & 14.89 & 10.84 \\
\hline \multicolumn{5}{|c|}{ VE } \\
\hline
\end{tabular}

Table 3. Means and Standard Deviations for Proportion of Errors, CE and VE for Dual-Task Tests

\begin{tabular}{|c|c|c|c|c|}
\hline \multicolumn{5}{|c|}{ Dual-task } \\
\hline \multirow[b]{2}{*}{ Test } & \multicolumn{2}{|c|}{ Self-Controlled } & \multicolumn{2}{|c|}{ Yoked } \\
\hline & $\mathbf{M}$ & SD & $\mathbf{M}$ & SD \\
\hline \multicolumn{5}{|c|}{ Proportion of Errors } \\
\hline I & .76 & .19 & .80 & .06 \\
\hline \multicolumn{5}{|c|}{$\mathbf{C E}$} \\
\hline I & 12.97 & 10.06 & 14.48 & 13.54 \\
\hline $\mathrm{D}$ & 17.62 & 13.02 & 16.89 & 11.96 \\
\hline \multicolumn{5}{|c|}{ VE } \\
\hline
\end{tabular}

Table 4. Means and Standard Deviations for Proportion of Errors, CE and VE for Transfer Tests

\begin{tabular}{|c|c|c|c|c|}
\hline \multicolumn{5}{|c|}{ Transfer } \\
\hline Test & $\mathbf{M}$ & SD & $\mathbf{M}$ & SD \\
\hline \multicolumn{5}{|c|}{ Proportion of Errors } \\
\hline I & .80 & .17 & .82 & .12 \\
\hline \multicolumn{5}{|c|}{$\mathbf{C E}$} \\
\hline $\mathrm{I}$ & 20.64 & 16.34 & 14.84 & 12.77 \\
\hline $\mathrm{D}$ & 21.94 & 16.33 & 24.52 & 14.33 \\
\hline \multicolumn{5}{|c|}{ VE } \\
\hline
\end{tabular}


from the home position. In general, participants in the selfcontrolled and yoked groups performed equally well at each target distance. The lack of differences between the selfcontrolled and yoked groups during acquisition was not unprecedented as several studies examining self-controlled versus yoked groups have not found benefits of self-controlled practice in acquisition (e.g. $[16,17])$. Self-controlled practice has been found to have a greater influence on measures of learning than on measures of performance [16, 17]. While measures of performance can provide an indication of the temporary influence of practice schedule type on performance, measures of learning dissociate the relatively permanent changes in the capability to perform the task (learning) [18].

\section{Measures of Learning}

The absence of differences between the self-controlled and yoked groups in both retention and transfer does not support our second hypothesis. Perhaps both groups experienced enough self-control in choosing how to propel the disc, that additional benefits were not seen for those that were also given the opportunity to choose the order of targets. Recent studies (e.g., [5]) suggest that it is the overall experience of self-control that is the beneficial factor during acquisition rather than the specifics of type of control $[5,16]$ or amount of control [19]. Neither group decreased in performance from acquisition to retention or in dual-task tests on the same target, suggesting that the groups experienced a mutual benefit rather than detriment during acquisition. Bund and Wiemeyer [5] explained that selfcontrol can create more demands on cognition and requires decision making as well as monitoring, evaluating and correction processes. Although overt decision-making during acquisition occurred when those in the self-controlled group chose the target for the next block of trials, each of the processes discussed by Bund and Wiemeyer [5] took place at the beginning of each trial for both the self-controlled and yoked groups. Both groups had control over hand positioning and force production on every trial and had the opportunity to make adjustments as needed. Thus, the added seven decisions as to the target presentation order provided to the self-controlled group may not have provided a benefit above and beyond the control used throughout practice. Bund and Wiemeyer [5] expanded the discussion of cognitive effort suggesting that strategies may make up a large portion of the cognitive effort taking place. In the case of the present experiment, though cognitive and informational strategies could be explored by choice of target presentation order, movement strategies could have been explored throughout acquisition. Wu and Magill [3] described a pre-determined schedule as inhibitory to choice, evaluation and exploration of strategies. This may be the case when participants are able to choose the order of individual trials, such as in $\mathrm{Wu}$ and Magill [3], however this may not hold true in the case of the present experiment where participants were only able to choose blocks of trials. In the present study participants may have made changes on a trial-by-trial basis to aspects of the task other than schedule, rather than on a block by block basis.
Another possible explanation for the lack of differences for the tests of learning is that the basic psychological needs of the learners in each group were met equally well [1]. Reeve [20] explained that along with the provision of choice, the provision of solid rationales, use of non-controlling language, acknowledgement of negative feelings and a patient approach can also promote feelings of autonomy and in turn influence behaviour. Since the researcher read the same instructions from a script to each of the participants and both groups were shown the test target at the beginning of practice, each of these additional factors should have been equated and may have led to both groups feeling that the need for autonomy was met. A limitation of the present study is that subjective experience in relation to the task was not measured.

\section{Measures of Implicit vs. Explicit Learning}

Regarding the third hypothesis, we predicted that the yoked group would experience decreased conscious processing of task information and decreased use of hypothesis testing strategies based on not being required to make decisions about target order. As discussed previously, participants from both groups may have in fact undertaken some type of cognitive effort. If this was the case, no differences in performance on the dual-task test would be expected.

Another way implicit learning, and therefore decreased conscious processing of task information and decreased hypothesis testing strategies, may have been introduced is through a pattern order strategy of targets that progressed from the easiest to the most difficult distance in order (or nearly so) [14]. Since half the participants in the selfcontrolled condition chose such a progressive schedule, and accordingly half the yoked participants followed a progressive schedule, it is interesting that neither a yoked practice condition nor a progressive pattern order strategy provided a benefit for performance on dual-task transfer tests. It is possible that a more implicit form of learning was not induced through either manipulation; however it is also possible that implicit learning was induced but was not effective in providing a benefit to performance on dual-task transfer tests.

\section{CONCLUSION}

The purpose of the present experiment was to determine if self-controlled and yoked practice schedules would elicit differences in a dual-task transfer test. The results did not reveal an influence of practice schedule on dual-task transfer test performance. Additionally, the results did not reveal an influence of practice schedule on retention or transfer tests. These surprising results highlight the intricacies of the effects of self-control and future studies should not only attempt to isolate the effects of self-control and yoked schedules when conducting dual task tests but should also explore the effects of layering self-control opportunities throughout practice. Measures of subjective experience in relation to the task would also be of benefit.

\section{CONFLICT OF INTEREST}

The authors confirm that this article content has no conflicts of interest. 


\section{ACKNOWLEDGEMENTS}

Funding for this research was provided by an NSERC Discovery Grant awarded to the second author.

\section{REFERENCES}

[1] Sanli EA, Patterson JT, Bray SR, Lee TD. Understanding selfcontrolled motor learning protocols through the self-determination theory. Front Psychol 2013; 3: 611

[2] Wulf, G. Self-controlled practice enhances motor learning: Implications for physiotherapy. Physiotherapy 2007; 93: 96-101.

[3] Wu W, Magill RA. Allowing learners to choose: Self-controlled practice schedules for learning multiple movement patterns. Res Q Exerc Sport 2011; 82: 449-57.

[4] Lewthwaite R, Wulf G. Motor learning through a motivational lens. In: Hodges NJ, Williams AM. Skill Acquisition in Sport: Research, Theory and Practice. $2^{\text {nd }}$ ed. New York: Routledge 2012; pp. 17391.

[5] Bund A, Wiemeyer J. Self-controlled learning of a complex motor skill: Effects of the learners' preferences on performance and selfefficacy. J Hum Mov Stud 2004; 47: 136-213.

[6] Keetch KM, Lee TD. The effects of self-regulated and experimenter-imposed practice schedules on motor learning for tasks of varying difficulty. Res Q Exerc Sport 2007; 78: 476-86.

[7] Brydges R, Carnahan H, Rose D, Dubrowski A. Comparing selfguided learning and educator-guided learning formats for simulation-based clinical training. J Adv Nurs 2010; 66: 1832-44.

[8] Hodges NJ, Edwards C, Luttin S, Bowcock A. Learning from the experts: Gaining insights into best practice during the acquisition of three novel motor skills. Res Q Exerc Sport 2011; 82: 178-87.

[9] Jowett N, LeBlanc V, Xeroulis G, MacRae H, Dubrowski A. Surgical skill acquisition with self-directed practice using computer-based video training. Am J Surg 2007; 193: 237-42.
[10] Post PG, Fairbrother JT, Barros JAC. Self-controlled amount of practice benefits learning of a motor skill. Res Q Exerc Sport 2011; 82: 474-81.

[11] Masters RSW. Knowledge, knerves and know-how: The role of explicit versus implicit knowledge in the breakdown of a complex motor skill under pressure. Br J Psychol 1992; 83: 343-58.

[12] Berry DC, Broadbent DE. Interactive tasks and the implicit-explicit distinction. Br J Psychol 1988; 79: 251-72.

[13] Baddeley AD, Wilson BA. When implicit learning fails: Amnesia and the problem of error elimination. Neuropsychologia 1994; 32: 53-68.

[14] Maxwell JP, Masters RSW, Kerr E, Weedon E. The implicit benefit of learning without errors. Q J Exp Psychol 2001; 54(A): 1049-68.

[15] Hancock GR, Butler MS, Fischman MG. On the problem of twodimensional error scores: Measures and analyses of accuracy, bias, and consistency. J Mot Behav 1995; 27: 241-50.

[16] Chen DD, Hendrick JL, Lidor R. Enhancing self-controlled learning environments: The use of self-regulated feedback information. J Hum Mov Stud 2002; 43: 69-86.

[17] Wulf G, Raupach M, Pfeiffer F. Self-controlled observational practice enhances learning. Res Q Exerc Sport 2005; 76: 107-11.

[18] Schmidt RA, Lee TD. Conditions of Practice. In: Schmidt RA, Lee TD, Eds. Motor Control and Learning: A Behavioral Emphasis. $5^{\text {th }}$ ed. Champaign: Human Kinetics 2011; pp. 347-91.

[19] Patterson JT, Carter M, Sanli E. Decreasing the proportion of selfcontrol trials during the acquisition period does not compromise the learning advantages in a self-controlled context. Res Q Exerc Sport 2011; 82: 624-33.

[20] Reeve J. Why teachers adopt a controlling motivating style towards students and how they can become more autonomy supportive. Educ Psychol 2009; 44(3): 159-75.

Received: July 10, 2013

Revised: August 06, 2013

Accepted: August 28, 2013

(C) Sanli and Lee; Licensee Bentham Open.

This is an open access article licensed under the terms of the Creative Commons Attribution Non-Commercial License (http://creativecommons.org/licenses/ by-nc/3.0/) which permits unrestricted, non-commercial use, distribution and reproduction in any medium, provided the work is properly cited. 